\title{
Wetland Information Extraction from RS Image Based on Wavelet Packet and the Active Learning Support Vector Machine
}

\author{
$\mathrm{Pu}$ Wang* and Wenxing Bao \\ School of Computer Science and Engineering, Beifang University for Ethnics, \\ Yinchuan Ningxia, P.R. China 750021 \\ flashstar521@yahoo.com.cn
}

\begin{abstract}
Wetlands which are the planet's most important ecosystem have high scientific research -value and will bring us both social and economic benefits. However, duing to various natural and man made factors, more and more wetlands have converted to agricultural land and urban land. Now, the changes in wetlands' area and quantity have caused public's widespread concern. And wetland's management and protection will benefit from the improvement of the wetland information abstraction's precision. Improving the classification precision of the RS image is a difficult problem because of the small scale of remote sensing images. This paper which is about the wetland remote sensing images extraction is based on the LANDSAT ETM remote sensing data, and the result of the Wavelet Packet reconstruction will be used as the sample set of the Active Support Vector Machine .At the end of this paper, a comparative analysis of the experimental results will show between the single classification (SVM, BPNN) method and the solution which is proposed in this article. This method can be proved to obtain very good classification results through many experiments on remote sensing image classification I've done. Experimental results show that this algorithm's classification accuracy is better than the single classification's. Moreover, in the active learning process, the bad influence of the image's isolated and intersection points on the classification is avoided, and the number of training samples are reduced greatly.
\end{abstract}

Keywords: Wavelet Packet, ASVM, wetland classification, remote sensing, information extraction.

\section{Introduction}

In recent years, with the development of RS technology, remote sensing images' classification methods which use traditional manual visual interpretation and parametric statistics have been difficult to meet the classification requirements of the multisource remote sensing data. Wetlands are usually situated between land and water, and traffic conditions there are very poor. Sometimes it is not easy to go deep into the

${ }^{*}$ Corresponding author.

D. Li, Y. Liu, and Y. Chen (Eds.): CCTA 2010, Part III, IFIP AICT 346, pp. 4914499, 2011.

(C) IFIP International Federation for Information Processing 2011 
wetlands field for survey. Therefore, extract wetland information with the help of RS data is very necessary [1]. There are a number of isolated points or noisy points in RS images, and the existence of these pixels severely affected the classification precision of wetlands from RS images. Therefore, we must find a method which can reduce the manual workload, speed up the computation, improve the wetlands' classification accuracy of remote sensing.

In the process of wetlands classification in remote sensing image, the artificial neural network, in particular BP neural network, has been relatively widely used. Neural network becomes a classifier through self-learning ability to obtain the weights. It is not a stable classifier and applicable to solve small data sets most of the time. Using BP algorithm to classification wetland in RS image requires long training time, and has a low convergence efficiency.

SVM (Support Vector Machine) was first proposed in 1995 by Corinna Cortes and Vapnik [2]. SVM is based on structure risk minimization principle to find the best compromise between the model's complexity and learning ability using the limitedsample information. And these limited samples called SV (Support Vector) which contain the necessary information in the classification. International experts have done a lot of research on SVM in remote sensing image for the wetland classification. This method can overcome the weakness of maximum likelihood method and neural network, and it suits the classification to non-linear, higher dimension and complex data. But in the process of sampling the remote sensing image using this method, the samples' manual labeled workload is heavy.

Wavelet packet algorithm provides a more precise and accurate processing analysis method for the signal frequency band. And non-support vector of SVM has no effect on classification. So, we can remove or reduce the non-SV from sample subset, and retain SV as much as possible .Therefore, the active support vector machine (ASVM) is derived. In order to solve the problems proposed in the above text, this paper based on MATLAB7.0 and ENVI4.4 software platform, using wavelet packet algorithm to preprocess remote sensing images, doing with the ASVM algorithm to realize the classification of wetlands, to improve these two problems in the RS image classification process in practical application. In recent years, ASVM's application gradually becomes research focus at home and abroad. Literature [3] proposed an active learning strategy of support vector machine via optimal selection of labeled data. And the workload of the sample tag was greatly reduced through the combination of unsupervised clustering method and active learning method. Literature [4] proposed a simple heuristic active support vector machines and it greatly improve the classification learning rate.

\section{Wavelet Packet Algorithm}

\subsection{The Definition of Wavelet Packet}

Wavelet packet analysis method is the promotion of multi-resolution wavelet analysis, simply, it is a function family. First, the two-scale relations of the orthogonal scaling function $\phi(t)$ and wavelet function $\psi(t)$ are as follows: 


$$
\begin{aligned}
& \phi(t)=\sqrt{2} \sum_{k} h_{0 k} \varphi(2 t-k) \\
& \psi(t)=\sqrt{2} \sum_{k} h_{1 k} \psi(2 t-k)
\end{aligned}
$$

( $h_{0 k}$ and $h_{1 k}$, the filter coefficients of multi-resolution analysis).

Promotion the two-scale equation above, define the following recurrence relation [5]:

$$
\begin{aligned}
& \omega_{2 n}(t)=\sqrt{2} \sum_{k \in z} h_{0 k} \omega_{n}(2 t-k), \\
& \omega_{2 n+1}(t)=\sqrt{2} \sum_{k \in z} h_{1 k} \omega_{n}(2 t-k),
\end{aligned}
$$

When $\mathrm{n}=0, \omega_{0}(t)=\phi(t)$; when $\mathrm{n}=1, \omega_{1}(t)=\psi(t)$.

The set of functions $\left\{\omega_{n}(t)\right\}, n \in z$ defined above is the Wavelet packet identified by the $\omega_{0}(t)=\phi(t)$. Thus, wavelet packet is a set that has some relation functions including the scaling function $\omega_{0}(t)$ and mother wavelet function $\omega_{1}(t)$.

\subsection{Wavelet Packet Decomposition Diagram}

Wavelet Packet Analysis can do further decomposed to high-frequency part. So, wetland's edge feature is enhanced through the Wavelet Packet decomposition and we can accurately find the RS image's effective information point which is easily overlooked. As shown in Figure 1, it is the diagram of wavelet packet decomposition.

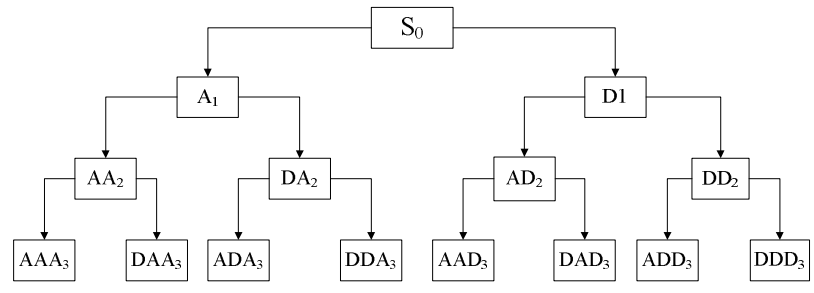

Fig. 1. Diagram of Wavelet Packet Decomposition

\section{Support Vector Machine}

\subsection{Support Vector Machine}

SVM [6] has been successfully applied in many areas, its main idea is to create a hyperplane as a decision surface that make positive samples and negative samples isolated between the maximize marginalized. As shown in Figure 2, it is the most simple linear separable classification hyperplane. 


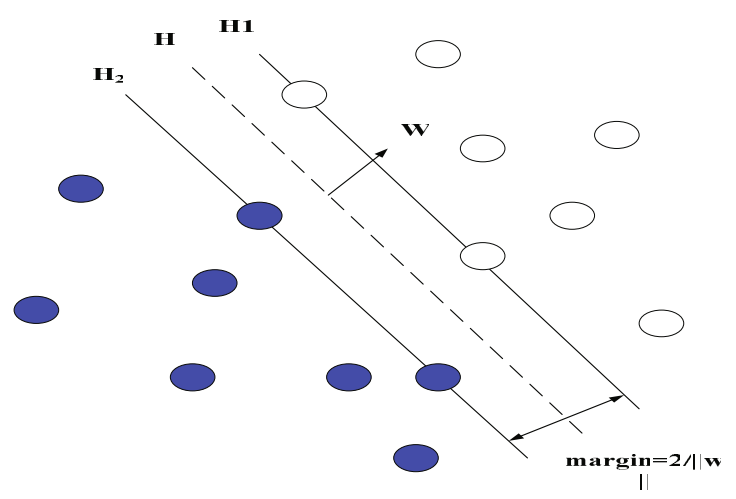

Fig. 2. Optimal Separating Surface (hyperplane) and SV

Generally, seeking the optimal hyperplane always through the establishment of Lagarange function and the introduction of Lagarange multiplier, that is, tansforming the original problem into the dual problem. As follows:

$$
\begin{gathered}
\max Q(\alpha)=\sum_{i=1}^{N} \alpha_{i}-\frac{1}{2} \sum_{i=1}^{N} \sum_{j=1}^{N} \alpha_{i} \alpha_{j} y_{i} y_{j} x_{i}^{T} x_{j} \\
\text { s.t } \sum_{i=1}^{N} \alpha_{i} y_{i}=0, \alpha_{i} \geq 0
\end{gathered}
$$

For nonlinear problems, the general solution is to use kernel functions which is satisfied Mercer conditions replacing the $\mathrm{xi}, \mathrm{xj}$ in the above equation. That is, using this kernel function to set the low-dimensional space vectors mapping in a highdimensional feature space, transforming the nonlinear problem into a linear problem.

\subsection{Active Support Vector Machine (ASVM)}

The main idea of ASVM: Selecting the most uncertain unlabeled samples (the samples nearest from the current hyperplane) from the candidate sets to improve the classification hyperplane.

\section{Experimental Data}

\subsection{The Area of Yinchuan Plain}

Climate in the Yinchuan Plain is a typical temperate continental climate. The main climatic characteristics: four distinct seasons, late spring and short summer, early autumn and long winter, large temperature difference between day and night, rare rain and snow, strong evaporation, dry climate, strong wind and more sand. Yinchuan Plain is the alluvial plain which is developed on the basis of the Cenozoic rift basin. Yellow River runs through Yinchuan plain vertically. Yinchuan Plain have a relatively 
abundant wetland resources which mainly including river wetlands and lake wetlands this two categories. The wetlands most locate at both sides of the Yellow River. Now, Yinchuan Plain's existing lakes have large shrinked surface and reduced water depth. And marsh plant community occupied considerable waters [7] of this area.

\subsection{Data Preparation}

As the remote sensing data is too large, a part of Yinchuan plain will test in this paper (area of Yinchuan plain in Yinchuan District). This article uses the Landsat ETM+ data in September 2006 as the basic data information. This year, the total rainfall in June to September (wet period) was lager than previous years and the vegetation coverage rate was higher too. So the data used in this paper has a better overall quality. The ETM+ is the Enhanced Thematic Mapper carried by Landsat satellite and its spatial resolution of reflectance band is $28.50 \mathrm{~m}$, panchromatic band is up to $14.25 \mathrm{~m}$. This thematic mapper applied for agricultural applications has relatively highly ground-based observations precision. On the basis of the research content, the researcher selects the present land-use map of Yinchuan Plain, the province administrative area map, traffic maps, soil maps, water conservancy maps of Ningxia in 2006 as auxiliary information. And the researcher makes a comprehensive analysis of the climate, topography, vegetation types and socio-economic factors of Yinchuan Plain to interpret remote sensing image for wetland.

\subsection{The Establishment of Yinchuan Plain's Information Classification System}

According to wetland classification standards that established by the National Survey of Wetland Resources and Procedures of Detection Technology and the actual situation of the Yinchuan Plain, based on the study purpose, this paper uses secondary classification standard to develop a wetland classification system which is consistent with national wetland classification requirements and can reflect the wetland characteristics of the region. As Table 1 shows:

Table 1. Wetland Interpretation Signs of Yinchuan Plain

\begin{tabular}{|c|c|c|c|c|}
\hline Type & Color & Shape & Distribution & Hue \\
\hline River/lake & blue & banded, block & natural & even \\
\hline River wetland & brownish-red & banded, curved & natural & even \\
\hline Lake wetland & yellow & block & natural & even \\
\hline swamp wetland & aquamarine & obvious & sporadic & uneven \\
\hline tidal flats & green & banded,curved & Coastal, along the lake & uneven \\
\hline pond & pink & obvious & natural & even \\
\hline
\end{tabular}

\subsection{RS Image Interpretation Signs of Yinchuan Plain}

The interpretation signs of the Yinchuan Plain established based on field survey and full use of the spectral reflectance characteristics of surface features and a variety of Yinchuan plain's maps and documents. As Table 1 shows: 


\section{Experimental Data Preprocessing and Algorithm Flow}

\subsection{Data Preprocessing}

Some bands' fusion of Lansat (band4, band5 and band3) not only fit for the analysis of soil moisture and vegetation but also suit for the identification of inland waters and land/water boundary. Therefore, data processing and information extraction in this paper mainly depend on these three bands. First of all, the researcher decomposites this three bands by wavelet packet algorithm in order to extract more refined timefrequency information, and then reconstructs the image and using the reconstructed results as the candidate sample set.

\subsection{Information Extraction by Active Learning Strategy}

In this paper, an unsupervised clustering method isodata is used to tag little samples to get a small amount of initial training sample set, and then using those taged samples to train a classifier. After the training, some strategies is utilized to re-updated the classification model, cycle update the classifier until the candidate set is empty or up to certain conditions.

The algorithm proposed in this paper is described as follows:

(1) Using DB2 wavelet and wavelet packet algorithm to decomposite the remote sensing image into 3-layer. And then, extracting each layer's all frequency components of the signal characteristics.

(2) Construction the optimal wavelet tree;

(3) Quantized each wavelet packet coefficients, and reconstructed the wavelet packet according to the lowest level wavelet packet decomposition coefficients and the quantized coefficient ;

(4) Take the reconstructed data set as a candidate sample set. Using Isodata algorithm to initially cluster the samples and producing $\mathrm{k}$ categories. Taking the centers of these $\mathrm{K}$ class as the initial training samples, denoted as A0, identified as labeled sample set. And the samples which are not selected is identified as unlabeled sample set, denoted as U0.

(5) Constructed the initial classifier. Using the sample set in step (4) to train SVM can obtain the initial classifier, the optimal hyperplane and support vectors.

(6) Finding the optimal separating hyperplane and support vector through the i-th active learning based on the labeled sample set, in the identification based on the sample set .Select the $\mathrm{m}$ sample points that are nearest from the optimal separating hyperplane, and then labeled these $\mathrm{m}$ sample points.

(7) Terminating the learning process when the unlabeled data set is empty or meet the required conditions. Otherwise, return step (6).

\section{Experimental Results and Analysis}

\subsection{Comparison of Experimental Results}

The 6 figures (Figure 3-8) below are the experimental results based on the algorithm proposed by this paper, SVM algorithm and BP neural network algorithm. Figure 4,6,8 


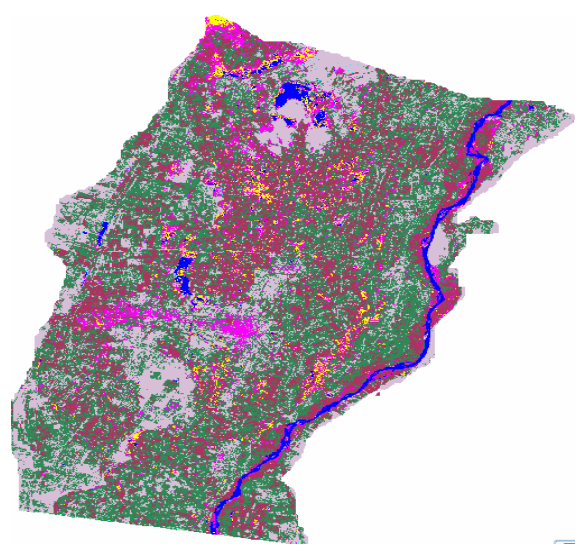

Fig. 3. Extraction results got by the algorithm proposed in this paper

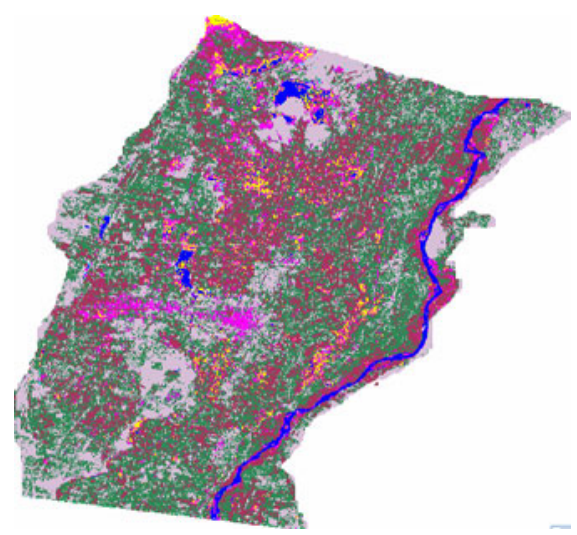

Fig. 5. Extraction results got by SVM algorithm

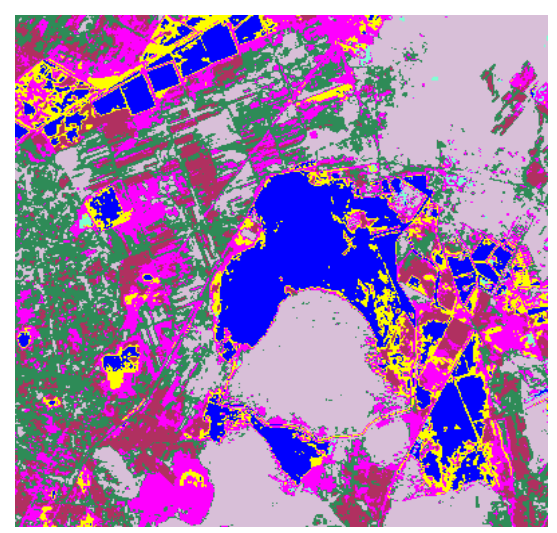

Fig. 4. Figure 4 Extraction results got by the algorithm proposed in this paper (Area of Shahu)

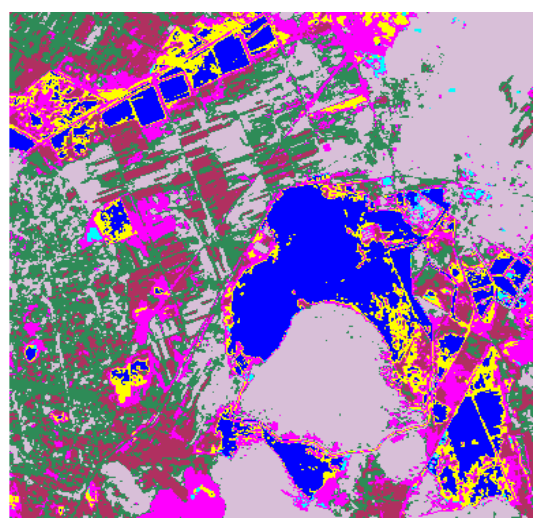

Fig. 6. Extraction results got by SVM algorithm (Area of Shahu)

are cut off from figure 3,5,7 and they display the area of Shahu in Yinchuan, so readers can clearly see the differences and advantages and disadvantages of experimental results.

\subsection{Results Analysis}

Through the 6 figures in chapter 5.1, we can see the classification results of every methods, and the results all have relatively clear boundaries, but have differences in the classification accuracy. Through the three pictures of Shahu area, it is obvious that the algorithm proposed in this paper and SVM algorithm get the similar classification results, and $\mathrm{BP}$ algorithm get a relatively rough classification result. The classification accuracy of this method is $83.39 \%$, SVM algorithm's accuracy is $82.14 \%$, and BP 


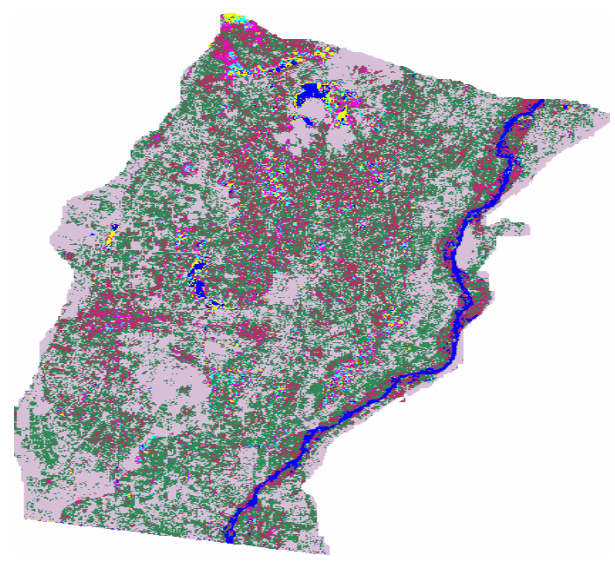

Fig. 7. Extraction results got by BP algorithm

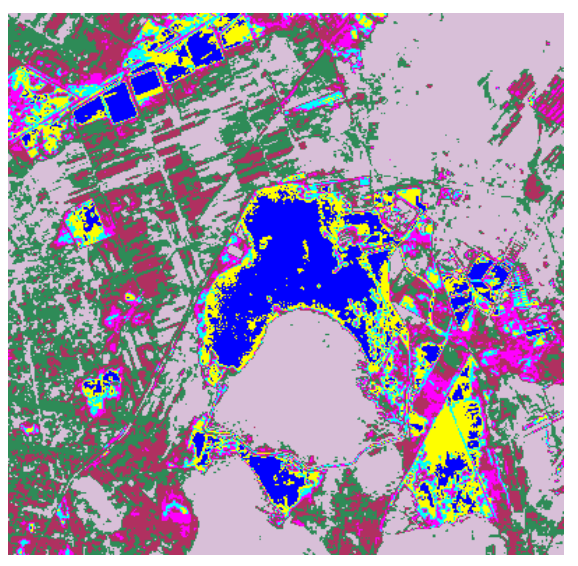

Fig. 8. Extraction results got by BP algorithm (Area of Shahu)

algorithm's classification accuracy is $74.63 \%$. This paper's algorithm is superior to SVM on the learning speed in classification and avoid a lot of manual work marked. Before the end of the experiment, the author and members of the research group made a surface sample survey (survey results are as follows in Table 2), in which this paper's algorithm and SVM algorithm both gets a high sampling precision and the precision is similar, but the BP algorithm gets a low sampling precision.

Table 2. Results of the surface sample survey

\begin{tabular}{|c|c|c|c|c|}
\hline \multirow{2}{*}{$\begin{array}{c}\text { Position } \\
\text { number }\end{array}$} & \multirow{2}{*}{$\begin{array}{c}\text { Actual } \\
\text { type }\end{array}$} & \multicolumn{3}{|c|}{ Classified type } \\
\cline { 3 - 5 } & & $\begin{array}{c}\text { This } \\
\text { algorithm }\end{array}$ & SVM & BP \\
\hline 1 & lake wetland & lake wetland & lake wetland & lake wetland \\
\hline 2 & swamp & swamp & swamp & tidal flats \\
\hline 3 & tidal flats & tidal flats & tidal flats & tidal flats \\
\hline 4 & lake wetland & lake wetland & lake wetland & lake wetland \\
\hline 5 & swamp & swamp & tidal flats & swamp \\
\hline 6 & pond & pond & swamp & pond \\
\hline 7 & lake wetland & lake wetland & lake wetland & lake wetland \\
\hline 8 & tidal flats & lake wetland & tidal flats & tidal flats \\
\hline 9 & river wetland & river wetland & river wetland & river wetland \\
\hline 10 & pond & pond & pond & swamp \\
\hline 11 & swamp & pond & pond & pond \\
\hline 12 & tidal flats & tidal flats & tidal flats & tidal flats \\
\hline 13 & river wetland & river wetland & river wetland & tidal flats \\
\hline 14 & lake wetland & lake wetland & lake wetland & lake wetland \\
\hline 15 & pond & pond & pond & pond \\
\hline
\end{tabular}




\section{Conclusion}

Using SVM to process the RS image needs large samples and will consume lots of manual labor, and the existed interferences of image noise, isolated points and the intersection points bring a lot of practical difficulties. To solve the above problems, we proposed an ASVM learning strategy. And it is eliminated the isolated points well, improved the accuracy of information classification, avoided a lot of manual marking work and solved the problem of slow classification rate in the process of learning.

Through the wetland information extraction of Yinchuan Plain in Yinchuan District, the results show that the degree of the artificial wetlands in the region is obvious and the natural wetlands are subjected to damage that we should take measures to strengthen the wetlands protection.

\section{Acknowledgements}

This work is supported by the National Key Technology R\&D Program of China under Grant NO. 2007BAD33B03.

\section{References}

[1] Sun, Y.: Study of Remote Sensing Mornitoring of Wetland Dynamic. PhD Thesis, 4 (2008)

[2] Vapnik, V.N.: The Nature of statistical learning theory, ch. 5. Wiley, New York (1998)

[3] Hu, Z.: An Active Learning Strategy of SVM Via Optimal Selection of Labeled Data. Signal Processing 24(1), 105-107 (2008) (Chinese)

[4] Chiu, W.-Y., Couloigner, I.: Modified fuzzy c-means classfication technique for mapping vague wetlands using Landsat ETM+ imagery. Hydrol. Process 20, 3623-3634 (2006)

[5] Ge, Z., Sha, W.: Wavelet analysis theory and Matlab R2007 achieve. Electronic Industry Press, Beijing (2007)

[6] Cristianini, N., Shawe-Taylor, J.: An Introduction to Support Vector Machines. Cambridge University Press, Cambridge (2000)

[7] Wetland protection regulations of Ningxia Hui Autonomous Region. Forestry Communications of Ningxia 3, 11-13 (2008) 